\title{
Active cancer and cardiac surgery: Possible scenarios in patient decision-making
}

\author{
Andrea Bonelli', Roberto Lorusso², Sara Paris', Giovanni Troise ${ }^{3}$, Abu Hilal Mohammed ${ }^{3}$, \\ Francesca Bursi ${ }^{4}$, Pompilio Faggiano ${ }^{1,3}$
}

${ }^{1}$ Cardiology Unit, Spedali Civili and University of Brescia, Italy; ${ }^{2}$ Department of Cardiothoracic Surgery, Heart and Vascular Centre, Maastricht University Medical Centre, Maastricht, The Netherlands; ${ }^{3}$ Fondazione Poliambulanza, Brescia, Italy; ${ }^{4}$ Division of Cardiology, Heart and Lung Department, San Paolo Hospital, ASST Santi Paolo and Carlo, University of Milan, Italy

\begin{abstract}
Heart disease and cancer are often found simultaneously in the same patient, and may require cardiac and non-cardiac surgery. Cancer may be part of the past medical history; in other cases the presence of an active malignancy makes the clinical management more complex. No general evidence-based recommendations are available to help in the decision-making process. Because of the lack of specific guidelines we provided a series of possible scenarios describing not unusual cases. We focused on cases where the concomitant presence of heart disease and active malignancies involved a multidisciplinary team. Four real patients with active cancer referred to our Center were assessed. Three of them had valve disease requiring cardiac surgery. Defining the timing of surgery and choosing the surgical approach required a careful and comprehensive evaluation. In the last case, the complicated bal-
\end{abstract} Correspondence: Dr. Pompilio Faggiano, Fondazione Poliambulanza,
Via Leonida Bissolati 57, Brescia, Italy.

E-mail: cardiologia@pompiliofaggiano.it

Authors contribution: $\mathrm{AB}, \mathrm{PF}$, conception, design and drafting of the manuscript; AB, SP, collected the patient data; RL, GT, FB, AM, revised it critically. All the authors have read and approved the final version of the manuscript and agreed to be accountable for all aspects of the work

Conflict of interest: The authors report no relationships that could be construed as a conflict of interest.

Key words: Cancer; cardiac disease; valve disease; cardiac surgery; oncologic surgery.

Received for publication: 25 October 2020.

Accepted for publication: 26 October 2020.

${ }^{\circ}$ Copyright: the Author(s), 2021

Licensee PAGEPress, Italy

Monaldi Archives for Chest Disease 2021; 91:1655

doi: 10.4081/monaldi.2021.1655

This article is distributed under the terms of the Creative Commons Attribution Noncommercial License (by-nc 4.0) which permits any noncommercial use, distribution, and reproduction in any medium, provided the original author(s) and source are credited. ance between the thrombotic and the hemorrhagic risk involved difficult decision. Several critical points, which characterized the management of this kind of patients, were identified. In particular, the hemodynamic status, the type and stage of the tumor, the need for cancer therapy, as well as the comorbidities of the patient, had to be taken into account. This narrative review shows the importance of submitting every challenging case to the assessment of a multidisciplinary team, which involves different clinical figures, in order to guarantee the most comprehensive evaluation. When clinical management deviates from the general recommendations, an individualized approach should be used.

\section{Introduction}

In current clinical practice, physicians are increasingly facing patients with active or remitted malignancies who require surgical or percutaneous cardiac interventions, whose evaluation needs multidisciplinary assessment and difficult decisions. Shared risk factors, ageing of population and increased life expectancy due to the improvement of diagnosis and treatment of cancer and cardiac disease are some of the reasons explaining this expanded simultaneous finding $[1,2]$.

Past history of cancer is reported in $1.9 \%$ to $4.2 \%$ of the patients referred to cardiac surgery [3]. It is known that this condition increases the risk of in-hospital complication after surgery because of multi-district structural abnormalities caused by previous exposition to radiation or chemotherapy $[3,4]$ However, data about mortality rates are conflicting, with different results according to the various settings. While some groups reported a significant impact of previous cancer on short and long-term mortality, other studies did not show any difference on outcomes $[5,6]$.

While history of cancer is not an absolute contraindication to cardiac surgery, in particular if the malignancy is on complete remission [6], on the other hand, surgical indication and operative risk assessment are challenging in case of active cancer: the optimal decision-making process is still matter of debate [1]. Some authors have already provided indications on how to approach these patients, highlighting those red-flags which must be taken into account [2].

In this narrative review, we focused on patients with active cancer presenting with a cardiac disease whose management might require surgical or other cardiac interventions. Some practical clinical scenarios, based on patients referred to our center, are presented, showing which critical issues have been assessed in the 
decision-making process, and how the management strategies have been supported by literature data.

\section{Scenario 1. Solid cancer and concomitant need for non-urgent mitral valve surgery: which first?}

The incidence rate of simultaneous heart disease and active cancer ranges between $3.4 \%$ and $7 \%$, with a higher proportion of solid malignancies over hematological $[7,8]$.

On February 2020, a 48-year-old male patient was referred because of a severe mitral regurgitation, as a consequence of infective endocarditis occurred two years earlier (Figure 1). He was also recently diagnosed with metastatic right colon adenocarcinoma, which was treated with neo-adjuvant chemo- and immunotherapy. Type 2 diabetes mellitus, bi-pulmonary transplant because of cystic fibrosis in 2009, previous thyroid and skin cancer and hemodialysis for a nephrotoxic disease represented associated comorbidities. Despite such a complicated medical history, he was in a good general clinical status, making the treatment of the cardiac abnormality advisable.

Even though this was an unusual case, some critical points made this an emblematic scenario. In fact, we had to confirm the surgical indications for both the cardiac and the cancer disease, and also to assess the operative risk and establish the surgical timing. While there are evidences for intervention and strategies for risk stratification, no general recommendations about operative timing are available.

The patient was completely asymptomatic, however the echocardiographic examination showed clear criteria for valve surgery according to international guidelines (LVEF $\leq 60 \%$, LVESD $\geq 45 \mathrm{~mm}$ ) [9]. Furthermore, the anatomical abnormalities of mitral valve made the surgical repair or transcatheter procedure unfeasible, making valve replacement the only possible solution.

Once valve surgery was confirmed, the patient was subjected to a complete oncologic assessment to stage the disease. He had a total body computed tomography $(\mathrm{CT})$, which did not find any sign of metastasis. Therefore, the patient resulted eligible for right colectomy, according to current guidelines [10]. In similar cases, long-term survival is influenced by both the heart and the cancer disease prognosis $[6,10]$. Their simultaneous presence seems to affect the surgical outcomes, increasing mortality after 24 months, due to tumor progression. On the other hand, medium-term survival do not differ and is acceptable [1].

The decision on the timing of the two interventions required a multidisciplinary-team discussion, including the cardiologist, the oncologist, the abdominal and the cardiac surgeons as well as the anesthesiologist.

One-stage surgery. In the past 30 years, several studies and case reports pointed out the feasibility of a one-stage surgical procedure in case of coronary artery by-pass grafting (CABG) [1114]. Only recently, this approach was employed for cardiac valve surgery. One-stage operation showed good early and mid-term outcomes. At the same time, long-term survival appears to be affected by malignancy: in fact, in several case series, late deaths were due to cancer progression [15]. The advantages of a simultaneous technique are attractive: one operation means a single exposition to anesthetic drugs and only one post-operative period, but it also means prolonged surgical time which, in case of reduced heart function and hemodynamic instability, could become fatal or predispose to perioperative complications. A potential better longterm outcome may be related to the absence of intervention delay and, of same importance, one-stage procedures have a better economic and psychosocial impact [16]. On the other hand, higher risk of hemorrhagic and infective complications makes this approach still not diffused. Some authors suggested that routine use of antibiotic prophylaxis, early extubation and, when feasible, avoidance of extracorporeal circulation (ECC) may be adequate ways to control complication occurrence [2,7].

To date, the one-step surgery is an acceptable option in selected cases: non-comorbid patients with good heart function, necessitating uncomplicated gastrointestinal resection [16].

Nevertheless, patients with concomitant heart and cancer disease usually undergo a two-stage intervention, based on clinical precedence. This technique is currently preferable because of the

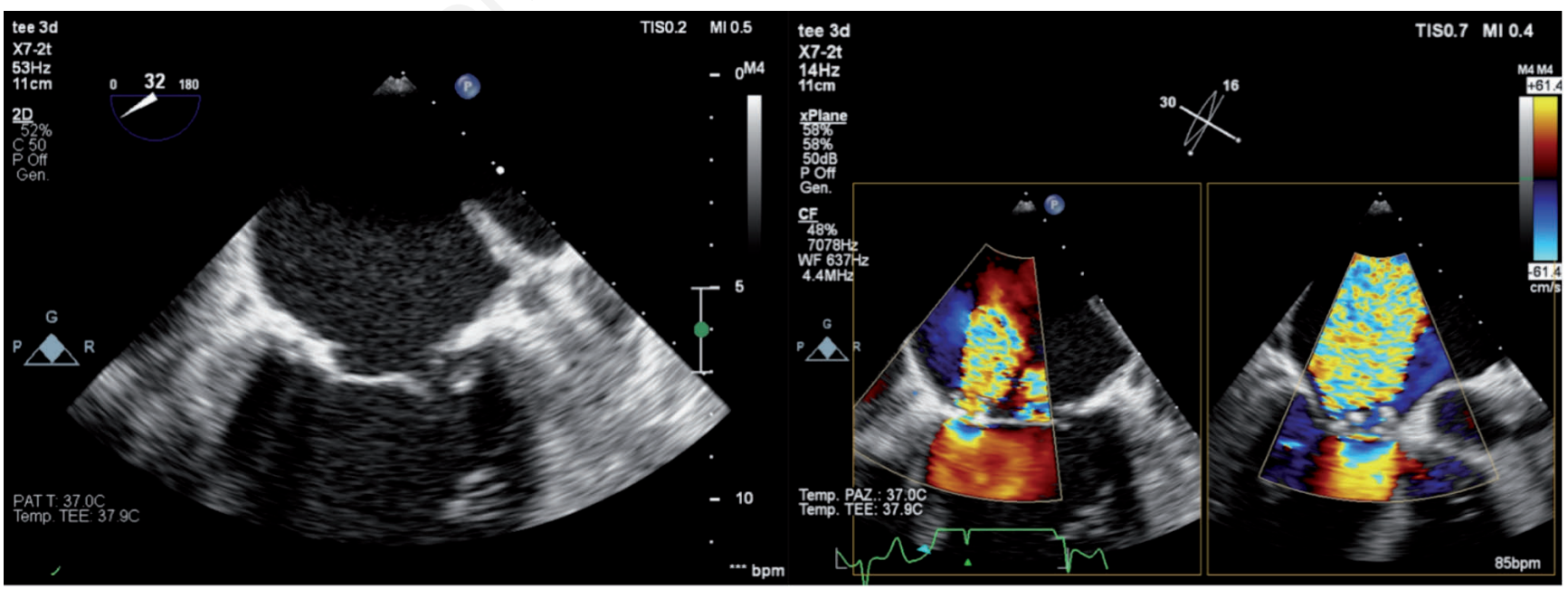

Figure 1. Transesophageal echocardiographic images of severe mitral regurgitation secondary to infective endocarditis. On the left, anatomical rearrangement of the mitral valve apparatus; note the abnormal coaptation of the mitral leaflets and the probable perforation. On the right, color Doppler flow imaging of the regurgitation; note the multiple jets in correspondence of the loss of substance. See the text for details. 
reduced hemorrhagic and infective risk, even if it forces the physicians to choose which disease should be treated first.

Therefore, because of the comorbidities of the patient, the multidisciplinary team opted for a two-step approach, instead of a simultaneous intervention. It was also decided to treat first the oncologic disease, thanks to the compensated hemodynamic status and the non-urgent need for mitral valve replacement. The ongoing immunosuppression, due to anti-rejection drugs, made the patient predisposed to severe infections and sepsis, particularly for the prosthetic valve to be implanted (Table 1).

The complex clinical background of the patient predicted a high post-operative morbidity and high risk of major complications [17-19]. He was also classified into class 4 of the American Society of Anesthesiologists Physical Status classification system (ASA PS) [20]. Thus, the high-risk profile affected the surgical and anesthesiologic planning.

In conclusion, patients with simultaneous heart disease and active cancer necessitate of careful multidisciplinary assessment because of the lack of strong recommendations. The available literature shows conflicting results, but it appears to lean towards a patient-centered approach. In this specific case, the confirmation of the tumor operability (absence of liver metastasis) and the deferrable intervention on mitral valve were the two critical points, which conditioned the operative choice.

\section{Scenario 2. Metastatic cancer and acute valve regurgitation: indication for surgery}

Assessing the prognosis of cancer disease is particularly crucial in patients presenting with acute valve failure due to infective endocarditis requiring urgent surgery.

On April 2020, a 72-year-old patient was hospitalized in septic shock. A multi-sensible Staphylococcus Aureus was isolated in the blood cultures, so specific antibiotic therapy was started. Due to the persistent hypotension and pulmonary congestion, the patient necessitated of vasopressor drugs (i.e. noradrenaline) and high furosemide doses. The echocardiography showed a severe acute aortic regurgitation due to leaflet destruction; thus, the cardiac surgeon was alerted. Over the following few days the clinical status slightly improved.

The patient medical history included prior operation for pancreatic cancer with lung metastasis, treated with chemotherapy and left lung lobectomy in 2016 (and a new lung lesion recently discovered), prior acute leukemia treated with chemotherapy in 2017, paroxysmal atrial fibrillation, chronic kidney disease, severe anemia and thrombocytopenia and type 2 diabetes.

Table 1. STS score for isolated mitral valve replacement (from STS Adult Cardiac Surgery Database Version 2.9). See the text for details.

\begin{tabular}{lc}
\hline Risk of mortality & $3.76 \%$ \\
Renal failure & $\mathrm{NA}^{*}$ \\
\hline Permanent stroke & $1.51 \%$ \\
Prolonged ventilation & $11.44 \%$ \\
\hline Morbidity or mortality & $19.35 \%$ \\
Short length of stay & $15.53 \%$ \\
\hline Long length of stay & $8.18 \%$ \\
\hline *Patient on hemodialysis. &
\end{tabular}

The operative risk was, therefore, high and challenging from the decision-making standpoint. The case was assessed by a multidisciplinary team. The oncologist considered the survival to be inferior to 1 year. Because of the poor prognosis and the clinical stabilization with medical therapy, the patient was judged to have no indication for cardiac surgery.

Cancer represents a frequent comorbidity in elderly patients requiring cardiac and non-cardiac interventions [21,22]. In these cases, the best approach is not well established because of the lack of evidence-based recommendations [23].

When compared to younger people, older patients often receive substandard treatment, partly owing to higher mortality and morbidity rates and shorter life expectancy. Thanks to the improvement of surgical and anesthesiologic techniques, access to surgery has gradually increased despite the high-risk conditions and the operative mortality has decreased [24]. Although surgical treatment appeared to be safe even in elderly people, care should be paid at the peri-operative management. It seems reasonable to assess every case by a multidisciplinary team which involves a geriatrician, in order to give a comprehensive geriatric assessment of comorbidities and functional status [25]. The preoperative assessment of cancer in the elderly (PACE) has been recently demonstrated to be a feasible tool to globally assess the cognitive and functional features of the elderly, prior to elective noncardiac surgery. Although this approach mainly refers to interventions for cancer disease, the authors highlighted the need for careful caseby-case evaluation to overcome the potential undertreatment [26].

Things are different when a patient with cancer disease requires cardiac surgery. In this case, the complex relationship between heart disease and cancer influences the therapeutic management.

First of all, before choosing the operative approach, the Heart Team should clarify cancer prognosis with an oncologist, including appropriate cancer staging [23]. While it is reasonable that previous or remitted malignancies are not considered as contraindication to surgery, the presence of active cancer is still matter of debate. Currently, patients with a favorable prognosis can be evaluated according to cardiovascular guidelines, while no general recommendations are available in case of worse prognosis. Moreover, in the former patients both surgical or transcatheter interventions can be performed. Recently, an algorithm was proposed to help clinicians in the management of severe aortic stenosis [23]: in case of prognosis of cancer disease unfavorable at short term (i.e. $<6$ months), only the medical palliative treatment is contemplated.

This case highlights that both cancer and cardiac valve prognosis, as well as the surgical urgency have influenced the therapeutic management. Infective endocarditis causing severe acute aortic valve regurgitation is known to have an unfavorable overall prognosis: in-hospital mortality ranges between $15 \%$ and $30 \%$. Renal impairment, S. Aureus bacteremia, heart failure and older age are predictors of poor outcome [27]. The ESC guidelines give clear indications for intervention: it is recommended that aortic endocarditis with sign of poor hemodynamic tolerance undergo urgent surgery (Class of Recommendation I, Level of Evidence B) [28,29].

Recently, it has been highlighted the lower long-term survival after urgent surgery compared to the elective one [30,31] However, surgery is still performed in more than the half of endocarditis patients, with a medium-term survival rates $>80 \%$. In real world setting, a significant proportion of patients do not undergo surgery, mainly because of pre-operative poor prognosis and hemodynamic instability. In typical cases, there are several scores which help the clinicians in risk assessment [32].

The present case was extensively discussed. The multidiscipli- 
nary team concurred on the avoidance of cardiac surgery because of the high risk of intervention, the poor neoplastic prognosis and the lack of available effective chemotherapy.

Once again, the optimal treatment should be established according to a case-by-case basis, especially when cancer is one of the comorbidities. After an accurate evaluation of the cardiac status and the indication for intervention, a complete cancer assessment should be performed. Specific account of the type of the cancer, its stage and the associated treatments is mandatory as it influences therapeutic choices. Not less important, the general functional status should be always globally assessed and quantified, possibly involving a geriatrician.

\section{Scenario 3. Active cancer and severe symptomatic aortic valve stenosis}

Because of the increased life expectancy of general population, cancer and aortic valve stenosis (AS) are common and can be found simultaneously. The actual prevalence of cancer in patients with severe aortic stenosis may exceed 25\% [33]. Not only age-dependency, but also shared predisposing factors, such as the common cardiovascular risk factors can explain this association [23].

On September 2016, a 78-year-old man was admitted for severe symptomatic aortic stenosis, with severe heart failure symptoms (New York Heart Association, NYHA, class III-IV). He did not have any previous medical contacts. The aortic valve area was $0.7 \mathrm{~cm}^{2}$, peak pressure gradient $68 \mathrm{mmHg}$, mean $43 \mathrm{mmHg}$, left ventricular ejection fraction $40 \%$, with moderate pulmonary hypertension (Figure 2). After medical stabilization, the preoperative coronary angiography showed a critical stenosis of the left anterior descendent artery. At the routine chest X-ray a lung nodule was detected; at the subsequent examination an isolated lung adenocarcinoma was diagnosed. The neoplasm was classified into an early stage, so that a surgical excision was considered feasible. The decision about the surgical planning was discussed in a multidisciplinary Team, which included an oncologist and a thoracic surgeon.

Current guidelines recommend multidisciplinary assessment of patients with severe aortic stenosis in order to choose the best operative approach: transcatheter (TAVR) or surgical aortic valve replacement (SAVR). In general, frail patients older than 75 years, with unfavorable anatomical characteristics and high surgical risk (assessed by STS or EuroSCORE II) should undergo TAVR. Conversely, indication in case of borderline patients is still challenging. Recent data suggest that there are some benefits in performing TAVR even in low-risk AS [34-36].

Managing cancer and aortic stenosis is not infrequent. Prior cancer treated with chemotherapy or radiotherapy often leads to valvular disease due to endothelial dysfunction and thickening or calcification of the valvular apparatus [37]. In some case series, more than $75 \%$ of patients who received radiation therapy can potentially have aortic valve calcification and stenosis [38].

In active cancer patients candidates to aortic valve replacement, recent data showed a major use of TAVR versus SAVR [39]. Patients undergoing TAVR were more likely to be younger, suggesting that the presence of cancer alone is widely considered a factor of worse prognosis. Indeed, one study showed a significantly high 1-year mortality in patient with active cancer undergoing TAVR [40,41]. Nevertheless, other authors found out similar mortality rates for TAVR and SAVR in cancer. Also, in case of transcatheter procedures there were less complications [39].

On contrast, recent literature provides conflicting results. One of the reasons is the lack of standardization for patient inclusion: the stage, the type of cancer and the age of the patient seems to affect the results. However, because of the increased perioperative risk [5] and the variety of clinical settings, SAVR might be an unsuitable solution to treat aortic stenosis in such kind of patients [40]. Of note, the mentioned surgical scores do not take into account active cancer stage, making the risk stratification arduous $[42,43]$.

Despite that, owing to a good functional status and the feasible less-invasive thoracic access (Video-assisted thoracoscopic surgery lobectomy), and because of the poor hemodynamic status of the valvular disease it was decided to perform the surgical aortic valve replacement combined with coronary artery by-pass grafting first (Table 2). So, tumor excision was performed later.

Lung cancer is the most frequent type of cancer preoperatively diagnosed in patients who need cardiac surgery. Recently, simultaneous interventions on lung and heart have been performed, not only in the CABG setting, but also for cardiac valves and thoracic aorta disease [15]. Concomitant treatment of cardiac and lung can-

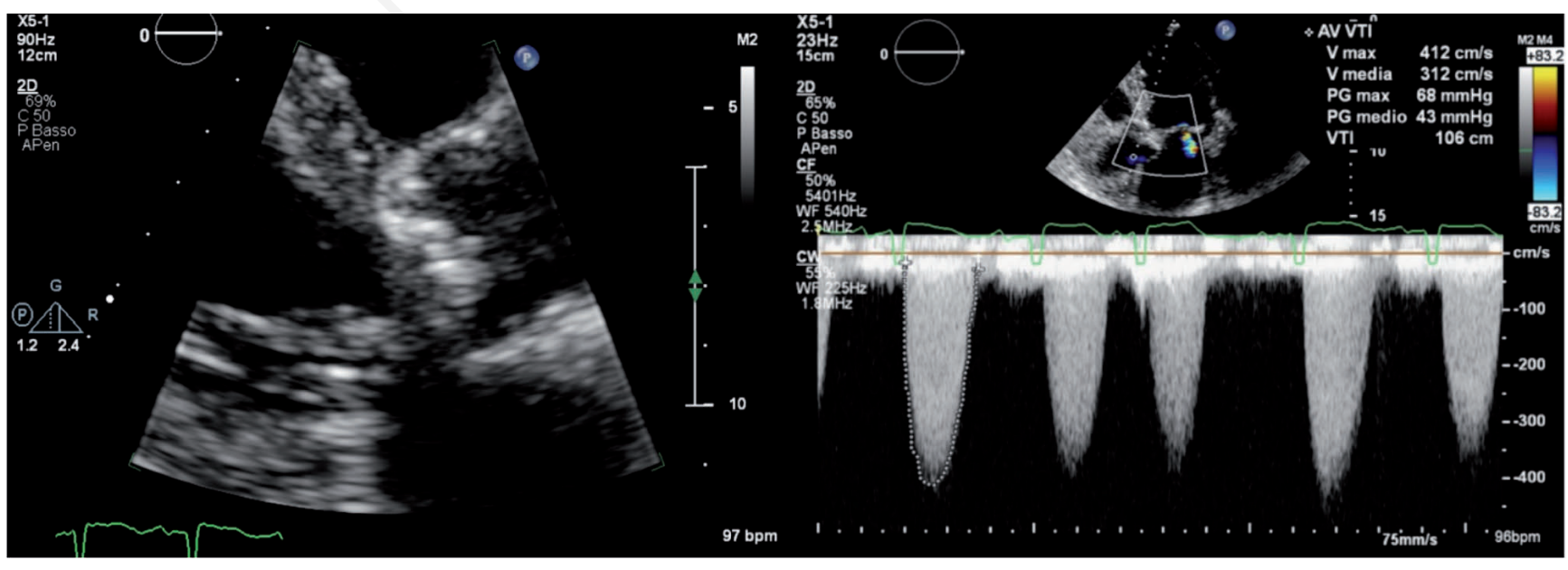

Figure 2. Transthoracic echocardiography of severe aortic valve stenosis. On the left the calcification of the aortic valve apparatus. On the right the highly elevated pressure gradient. 
cer disease was demonstrated to be safe and effective in selected patients, especially those with Stage I or II pulmonary malignancies [44-47]. One study confirmed the feasibility of the simultaneous approach without evidence of severe complications [15]. Nevertheless, caution must be used in case of poor hemodynamic status or technically demanding procedures. A 2-stage approach appears to be safer [48]

Of interest, even though plausible, the association between cardiopulmonary by-pass (CPB) and cancer progression do not appear to be supported by evidence-based data [45]. While few authors reported a lower incidence of cardiac death versus non-cardiac mortality, particularly related to cancer (7\% vs 21\%) [49], many groups demonstrated no significant impact of CBP on cancer progression during a long-term follow-up [46]. Certainly, surgical stress was ascertained to affect the immune system, resulting in increasing tumor recurrence. This may be the pathogenetic cause which explains CBP and cancer relation [50]. To date, CBP is not likely to cause tumor progression.

In conclusion, we provided an example of challenging, but not unusual, decision-making process. It seems reasonable that the pre-operative global assessment of the patient with active malignancy should be individualized. Any comorbidities and potential toxic effects of chemotherapy, in particular on the hematological field, should be taken into account in risk stratification [51].

Table 2. STS score for aortic valve replacement and coronary artery bypass grafting (from STS Adult Cardiac Surgery Database Version 2.9). See the text for details.

\begin{tabular}{lc}
\hline Risk of mortality & $1.79 \%$ \\
Renal failure & $1.62 \%$ \\
\hline Permanent stroke & $1.43 \%$ \\
Prolonged ventilation & $5.39 \%$ \\
\hline Morbidity or mortality & $10.69 \%$ \\
Short length of stay & $36.06 \%$ \\
\hline Long length of stay & $5.59 \%$ \\
\hline
\end{tabular}

Active cancer is not an absolute contraindication to surgery, especially if it is at an initial stage and it is the only comorbidity of a patient. Further investigations will clarify which diagnostic-therapeutic process is the best for this kind of patients. Simultaneous interventions should be performed in selected patients and, probably, in dedicated centers.

\section{Scenario 4. Managing hemorrhagic and thrombotic risk in active cancer: left atrial appendage exclusion}

Both cancer disease and antineoplastic drugs affect human coagulation system. The raised incidence of thromboembolic complications, as much as hemorrhagic ones, leads to the need for balancing of the two risks in routine clinical practice [2].

On March 2020, a 64-year-old man with a past history of paroxysmal atrial fibrillation (AF) and a recent recurrence of ischemic stroke came to our attention. The transesophageal echocardiogram (TEE) showed a left atrial appendage thrombus (Figure 3). Although he was on direct oral anticoagulant (DOAC) therapy for stroke prevention, the patient was recently asked to stop DOAC because of the appearance of pericardial effusion with cardiac tamponade. In fact, the pericardiocentesis showed a hematic fluid with neoplastic cells. The cells were characterized as diffuse large B-cell lymphoma, according to WHO classification [52], and the positron emission tomography classified the neoplasm in an advanced stage (stage IV) [53].

Thus, given the urgent need for starting lymphoma chemotherapy, the assessment and the management of the thromboembolic risk involved a multidisciplinary team. Some international societies have provided guidelines, but the complex background of some patients often makes the decision challenging [54,55].

The interaction between cancer and coagulation disorders is complex. Both solid and hematological cancers predispose the patient to a various spectrum of thrombotic or hemorrhagic events, from venous or arterial thrombosis phenomena to life-threatening hemorrhages and disseminated intravascular coagulation (DIC). Thrombosis occurrence is the best described topic in literature: in
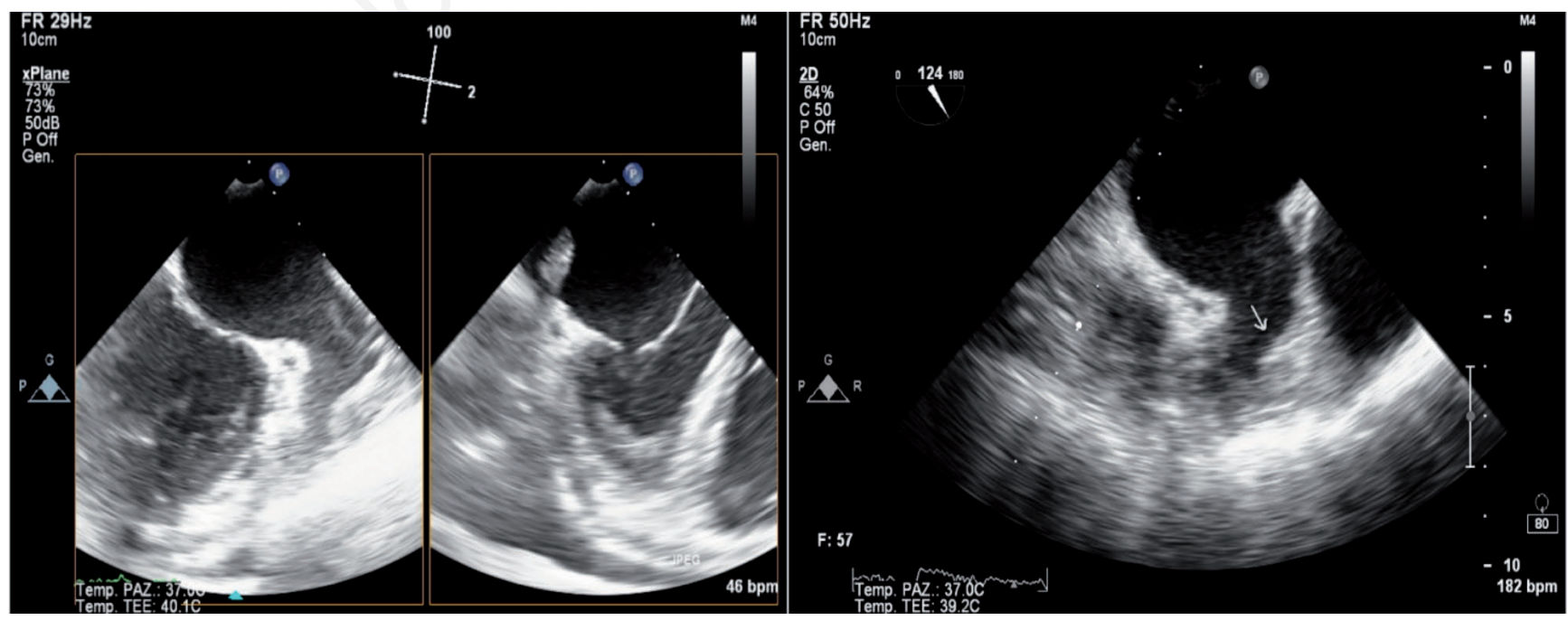

Figure 3. Left atrial appendage thrombus. Left. At admission the thrombus occupied the cavity. Right. Note the reduced dimension of the thrombus after 10 days of unfractionated heparin therapy (white arrow). See the text for details. 
different case series, the incidence of venous thromboembolism (VTE) in cancer ranges from $0.6 \%$ to $7.8 \%$, with deep venous thrombosis as the most frequent, followed by pulmonary embolism [56]. On the other hand, arterial thrombosis occurrence appears to have an inferior incidence (0\%-1.6\%) [57-59].

In cancer patients, many risk factors may contribute to increase thromboembolic events. They can be categorized into three groups: patient-related, cancer-related and treatment-related, as summarized in Table $3[60,61]$. Next to the general risk factors shared with noncancer patients, also the type of cancer affects thrombosis occurrence. Pancreatic, hematological, lung and brain malignancies carry the highest risk [62-64]. As for cancer treatment, doxorubicin and dexamethasone use determines a high pro-thrombotic profile [65]. In addition, atrial fibrillation is relatively common in cancer patients and requires thromboembolic prophylaxis.

The other side of the coagulopathy spectrum is characterized by hemorrhages. Although bleeding disorders are more uncommon, about $10 \%$ of both solid and hematological cancer patients experiment hemorrhagic conditions, with a consistent impact on mortality [66]. The occurrence of severe DIC is particularly high in case of acute leukemias; one study has recently shown a $7 \%$ incidence of DIC in solid tumors, too [67]. In general, people with cancer are at two- to six-fold increased risk of hemorrhages. The incidence of bleeding disorders raises in case of pre-existing risk factors, many of which are the same as thrombotic ones [2]. Precisely, thrombocytopenia, severe liver disfunction or vitamin $\mathrm{K}$ deficiency causing reduced synthesis of coagulation factors, oral anticoagulation therapy or some inherited clotting factor deficiencies may affect hemorrhagic risk [60].

Of interest, acquired hemophilia should be suspected in cancer patients with unexplained predisposition to bleeding. Its presence is cause of considerable morbidity and mortality [66]. From the pathogenetic point of view, cancer cells can interfere with coagulation at different levels. The best recognized pro-coagulant cancer-related factor is tissue factor (TF). Its overexpression leads to a hypercoagulable state which predisposes to both central and peripheral thrombosis [2]. Also, another factor produced by cancer cells, called cancer procoagulant, leads to the same predisposition $[68,69]$. Tumors can affect both clotting and fibrinolytic cascade and they promote cytokine release, cyclooxygenase-2 (COX-2) activation, leucocyte adhesion and endothelial cell disfunction [60].

Although not validated for cancer patients, AF-related hemorrhagic and thrombotic risk using CHA2DS2-VASc and HASBLED scores, as recommended by current guidelines, were assessed in this patient [70]. Both scores (CHA2DS2-VASc $=3$; HAS-BLED=3) identified a high risk of thromboembolic and hemorrhagic complications. However, the multidisciplinary team was inclined to avoid long-term anticoagulation therapy because of the history of severe life-threatening hemorrhagic condition (i.e., cardiac tamponade) and the need for chemotherapy potentially acting on the coagulation system. So, left atrial appendage (LAA) occlusion for stroke prevention was considered before lymphoma treatment [70]. Two randomized controlled trials demonstrated the non-inferiority to warfarin of percutaneous LAA occlusion with WATCHMAN left atrial appendage closure device (Boston Scientific, Marlborough, MA, USA). Also, the successful rate is very high $[71,72]$. On contrast, the post-procedural need for dual antiplatelet therapy (DAPT) or dual therapy with anticoagulant and single antiplatelet drug made the procedure still unfeasible in some cases [73].

Based on these considerations, after the partial resolution of LAA thrombus with short-term intravenous heparin administration (without hemorrhagic complications), the patient underwent surgical thoracoscopic epicardial exclusion of LAA. The intervention was performed without complication. During the following days, the patient received prophylaxis therapy (enoxaparin $40 \mathrm{mg}$ once a day) due to the high post-surgical thrombotic risk. Eventually, the multidisciplinary team concurred on maintaining single antiplatelet therapy.

There are not well-defined recommendations about thromboprophylaxis in cancer patients. In the arterial ischemia setting, some data suggest the use of aspirin for primary and secondary prevention $[74,75]$. In particular, antiplatelet therapy showed a significant reduction of cardiovascular events in case of myeloproliferative neoplastic disease [76]. The only approved drug for primary VTE-prevention in cancer patients is low-molecular weight heparin (LMWH). While all hospitalized solid cancer patients should receive LMWH prophylactic dose [77], in the outpatient setting, routine use of thromboprophylaxis is not recommended, although feasible and safe [78]. Unlike solid tumors, data about hematological malignancies are lacking.

In general, recent guidelines highlight the need for individualized risk assessment [79]. Some risk models are available but still not validated [80].

In conclusion, therapeutic and interventional choices, in this case, were affected by the type and stage of cancer disease and the clinical background of the patient. Due to the complexity of the clinical setting and the lack of strong recommendations, a multidisciplinary team was involved. Balancing thromboembolic and hemorrhagic risk in such kind of patients is challenging and it requires careful overall evaluation. Also, because of the cardiotoxic effect of chemotherapy drugs, a close cardiologic follow-up must be provided for these patients.

Table 3. Risk factors for VTE in cancer patients.

\begin{tabular}{lll}
\hline Patient-related [69] & Cancer-related & Treatment-related \\
Age ( $>60$ yrs) & Site of cancer (pancreas, hematologic, lung, brain) & Hospitalization \\
Gender (female) & Stage of cancer: advanced stage and initial period & ASA class \\
Race (Africa-Americans) & after diagnosis [75] & Surgery \\
Performance status & & Chemotherapy \\
Immobility & & Hormonal therapy \\
BMI & Anti-angiogenic therapy \\
Prior thrombotic event & Erythropoiesis stimulating agents \\
Inherited prothrombotic mutations & Blood transfusions \\
Comorbidities (infection, pulmonary disease, & Central venous catheter (presence and site) [76,77] \\
renal disease) & Radiations \\
\hline
\end{tabular}




\section{Conclusions/Take-home messages}

Nowadays, the number of patients requiring cardiac interventions who are simultaneously affected by cancer disease is increasing. Sometimes patients present themselves with previous diagnosis of cancer, but tumor is often diagnosed during the pre-operative assessment. In case of active malignancies, owing to the lack of standard recommendation, physicians should take a case-by-case approach. This becomes considerable in order to avoid both overand undertreatment.

In our report we highlighted some critical points, which can be summarized as below:

- Definition of etiology, indications for surgery and hemodynamic status of the cardiac disease.

- Definition of type, stage and prognosis of the active cancer disease.

- Pre-operative global assessment of the functional status of the patient.

- Stratification of thrombotic or hemorrhagic risk.

- Timing of surgery based on clinical priority: 2-stage or 1-stage intervention.

- Type of surgical approach (i.e. use of extracorporeal circulation).

- Need for pre- or post-operative chemo- or radiotherapy.

To obtain a comprehensive evaluation, a dedicated HeartCancer Team is necessary; it should always involve an oncologist and a geriatrician, because of the great proportion of elderly among these specific kinds of patients.

In light of the showed preliminary mortality results, further investigations and randomized controlled trials are required to clarify the recommendations, the benefits and the optimal approach in these specific cases.

\section{References}

1. Carrascal Y, Gualis J, Arévalo A, et al. Cardiac surgery with extracorporeal circulation in cancer patients: Influence on surgical morbidity and mortality, and on survival. Rev Española Cardiol (English Ed. 2008). doi: 10.1016/s1885-5857(08) 60137-1

2. Lorusso R, Vizzardi E, Johnson DM, et al. Cardiac surgery in adult patients with remitted or active malignancies: A review of preoperative screening, surgical management and short-and long-term postoperative results. Eur J Cardio-thoracic Surg 2018. doi: 10.1093/ejcts/ezy019

3. Cheng H, Force T. Molecular mechanisms of cardiovascular toxicity of targeted cancer therapeutics. Circ Res 2010. doi: 10.1161/CIRCRESAHA.109.206920

4. Yeh ETH, Bickford CL. Cardiovascular complications of cancer therapy. J Am Coll Cardiol 2009. doi: 10.1016/j.jacc.2009. 02.050

5. Chan J, Rosenfeldt F, Chaudhuri K, Marasco S. Cardiac surgery in patients with a history of malignancy: Increased complication rate but similar mortality. Hear Lung Circ 2012. doi: 10.1016/j.hlc.2012.02.004

6. Mistiaen WP, Van Cauwelaert P, Muylaert P, et al. Effect of prior malignancy on survival after cardiac surgery. Ann Thorac Surg 20047. doi: 10.1016/j.athoracsur.2003.08.049

7. Eagle KA, Ginsburg GS, Musunuru K, et al. Identifying patients at high risk of a cardiovascular event in the near future: Current status and future directions: Report of a national heart, lung, and blood institute working group. Circulation 2010. doi: 10.1161/CIRCULATIONAHA.109.904029

8. Wu W, Masri A, Popovic ZB, et al. Long-term survival of patients with radiation heart disease undergoing cardiac surgery: A cohort study. Circulation 2013. doi: 10.1161/CIRCULATIONAHA.113.001435

9. Baumgartner H, Falk V, Bax JJ, et al. 2017 ESC/EACTS Guidelines for the management of valvular heart disease. Eur Heart J 2017. doi: 10.1093/eurheartj/ehx391

10. Benson AB, Venook AP, Al-Hawary MM, et al. NCCN guidelines insights: Colon cancer, version 2.2018. J Natl Compr Cancer Netw 2018. doi: 10.6004/jnccn.2018.0021

11. Kirchmeyer M, Kalweit G, Gams E. Extended left pneumonectomy combined with off-pump coronary revascularization (CABG). Thorac Cardiovasc Surg 2000. doi: 10.1055/s-20006889

12. Dyszkiewicz W, Jemielity MM, Piwkowski CT, et al. Simultaneous lung resection for cancer and myocardial revascularization without cardiopulmonary bypass (off-pump coronary artery bypass grafting). Ann Thorac Surg 2004. doi: 10.1016/j.athoracsur.2003.07.041

13. Davydov MI, Akchurin RS, Gerasimov SS, et al. Simultaneous operations in thoraco-abdominal clinical oncology. Eur J Cardio-Thoracic Surg 2001. doi: 10.1016/S1010-7940(01) 00922-8

14. Mariani MA, Van Boven WJ, Duurkens VAM, et al. Combined off-pump coronary surgery and right lung resections through midline sternotomy. Ann Thorac Surg 2001. doi: 10.1016/S0003-4975(00)02355-9

15. Fu Q, Li QZ, Liang DG, et al. Early and long-term results of combined cardiac surgery and neoplastic resection in patients with concomitant severe heart disease and neoplasms. Chin Med J (Engl) 2011. doi: 10.3760/cma.j.issn.0366-6999.2011.13.004

16. Komokata T. Simultaneous operation for cardiac disease and gastrointestinal malignancy. World J Gastrointest Surg 2014. doi:1 $0.4240 /$ wjgs.v6.i8.146

17. Coimbra FJF, de Jesus VHF, Franco CP, et al. Predicting overall and major postoperative morbidity in gastric cancer patients. J Surg Oncol 2019. doi: 10.1002/jso.25743

18. Pandit V, Khan M, Martinez C, et al. A modified frailty index predicts adverse outcomes among patients with colon cancer undergoing surgical intervention. Am J Surg 2018. doi: 10.1016/j.amjsurg.2018.07.006

19. Iyengar A, Goel N, Kelly JJ, et al. Effects of frailty on outcomes and 30-day readmissions after surgical mitral valve replacement. Ann Thorac Surg 2020. doi: 10.1016/j.athoracsur.2019.10.087

20. Hackett NJ, De Oliveira GS, Jain UK, Kim JYS. ASA class is a reliable independent predictor of medical complications and mortality following surgery. Int J Surg 2015. doi: 10.1016/j.ijsu. 2015.04.079

21. Alexander HR, Turnbull AD, Salamone J, et al. Upper abdominal cancer surgery in the very elderly. J Surg Oncol 1991. doi: $10.1002 /$ jso. 2930470205

22. Ahlgren JD. Gastrointestinal cancer in the elderly. Clin Geriatr Med 1999. doi: 10.1016/s0749-0690(18)30171-x

23. Balanescu SM, Balanescu DV, Donisan T, et al. The onco-cardiologist dilemma: to implant, to defer, or to avoid transcatheter aortic valve replacement in cancer patients with aortic stenosis? Curr Cardiol Rep 2019. doi: 10.1007/s11886-019-1166-0

24. Greenfield LJ. Surgery of the aged. Management of respiratory problems. Major Probl Clin Surg 1975. 
25. Stuck AE, Siu AL, Wieland GD, et al. Comprehensive geriatric assessment: a meta-analysis of controlled trials. Lancet 1993. doi: 10.1016/0140-6736(93)92884-V

26. PACE Participants, Audisio RA, Pope D, et al. Shall we operate? Preoperative assessment in elderly cancer patients (PACE) can help. A SIOG surgical task force prospective study. Crit Rev Oncol Hematol 2008 doi: 10.1016/j.critrevonc.2007.11.001

27. Habib G, Lancellotti P, Antunes MJ, et al. 2015 ESC Guidelines for the management of infective endocarditis. Eur Heart J 2015. doi: 10.1093/eurheartj/ehv319

28. López J, Sevilla T, Vilacosta I, et al. Clinical significance of congestive heart failure in prosthetic valve endocarditis. A multicenter study with 257 patients. Rev Española Cardiol 2013. doi: 10.1016/j.rec.2012.10.022

29. Habib G, Tribouilloy C, Thuny F, et al. Prosthetic valve endocarditis: Who needs surgery? A multicentre study of 104 cases. Heart 2005. doi:10.1136/hrt.2004.046177

30. Musci M, Weng Y, Hübler M, et al. Homograft aortic root replacement in native or prosthetic active infective endocarditis: Twenty-year single-center experience. J Thorac Cardiovasc Surg 2010. doi: 10.1016/j.jtcvs.2009.07.026

31. Manne MB, Shrestha NK, Lytle BW, et al. Outcomes after surgical treatment of native and prosthetic valve infective endocarditis. Ann Thorac Surg 2012. doi: 10.1016/j.athoracsur.2011.10.063

32. Cahill TJ, Baddour LM, Habib G, et al. Challenges in Infective Endocarditis. J Am Coll Cardiol 2017. doi: 10.1016/j.jacc. 2016.10.066

33. Faggiano P, Frattini S, Zilioli V, et al. Prevalence of comorbidities and associated cardiac diseases in patients with valve aortic stenosis. Potential implications for the decision-making process. Int J Cardiol 2012. doi: 10.1016/j.ijcard.2011.02.026

34. Freemantle N, Irs A, De Paulis R, et al. Transcatheter versus surgical aortic valve replacement: What does the latest evidence tell us? Eur J Cardio-thoracic Surg 2019. doi: 10.1093/ejcts/ezz163

35. Popma JJ, Michael Deeb G, Yakubov SJ, et al. Transcatheter aortic-valve replacement with a self-expanding valve in lowrisk patients. N Engl J Med 2019. doi: 10.1056/NEJMoa 1816885

36. Mack MJ, Leon MB, Thourani VH, et al. Transcatheter aorticvalve replacement with a balloon-expandable valve in low-risk patients. N Engl J Med 2019. doi: 10.1056/NEJMoa1814052

37. Donnellan E, Griffin BP, Johnston DR, et al. Rate of progression of aortic stenosis and its impact on outcomes in patients with radiation-associated cardiac disease: A matched cohort study. JACC Cardiovasc Imaging 2018. doi: 10.1016/j.jcmg. 2018.04.019

38. Darby SC, Ewertz M, McGale P, et al. Risk of ischemic heart disease in women after radiotherapy for breast cancer. N Engl J Med 2013. doi:10.1056/NEJMoa1209825

39. Guha A, Dey AK, Arora S, et al. Contemporary trends and outcomes of percutaneous and surgical aortic valve replacement in patients with cancer. J Am Heart Assoc 2020. doi: 10.1161/ JAHA. 119.014248

40. Schechter M, Balanescu DV, Donisan T, et al. An update on the management and outcomes of cancer patients with severe aortic stenosis. Catheter Cardiovasc Interv 2019. doi: 10.1002/ccd.28052

41. Mangner N, Woitek FJ, Haussig S, et al. Impact of active cancer disease on the outcome of patients undergoing transcatheter aortic valve replacement. J Interv Cardiol 2018. doi: $10.1111 /$ joic. 12458
42. Nashef SAM, Roques F, Michel P, et al. European system for cardiac operative risk evaluation (EuroSCORE). Eur J Cardiothoracic Surg 1999. doi: 10.1016/S1010-7940(99)00134-7

43. Shroyer ALW, Coombs LP, Peterson ED, et al. The society of thoracic surgeons: 30-Day operative mortality and morbidity risk models. Ann Thorac Surg 2003. doi: 10.1016/S00034975(03)00179-6

44. Danton MHD, Anikin VA, McManus KG, et al. Simultaneous cardiac surgery with pulmonary resection: Presentation of series and review of literature. Eur J Cardio-thoracic Surg 1998. doi: 10.1016/S1010-7940(98)00088-8

45. Negaard HFS, Iversen PO, Østenstad B, et al. Increased acquired activated protein $\mathrm{C}$ resistance in unselected patients with hematological malignancies. J Thromb Haemos. 2008. doi: 10.1111/j.1538-7836.2008.03060.x

46. Habib RH, Zacharias A, Engoren M. Determinants of prolonged mechanical ventilation after coronary artery bypass grafting. Ann Thorac Surg 1996. doi: 10.1016/00034975(96)00565-6

47. Moshkovitz Y, Mohr R. Coronary artery bypass without cardiopulmonary-bypass - The pros and the cons. Isr J Med Sci 1993;29):716-20.

48. Tourmousoglou CE, Apostolakis E, Dougenis D. Simultaneous occurrence of coronary artery disease and lung cancer: what is the best surgical treatment strategy? Interact Cardiovasc Thorac Surg 2014. doi: 10.1093/icvts/ivu218

49. Vieira RD, Pereira AC, Lima EG, et al. Cancer-related deaths among different treatment options in chronic coronary artery disease: Results of a 6-year follow-up of the MASS II study. Coron Artery Dis 2012. doi: 10.1097/MCA.0b013e32834f112a

50. Braile DM, Évora PRB. Cardiopulmonary bypass and cancer dissemination: A logical but unlikely association. Brazilian J Cardiovasc Surg 2018. doi: 10.21470/1678-9741-2018-0600

51. Zhu Y, Toth AJ, Lowry AM, et al. Cardiac surgery outcomes in patients with chronic lymphocytic leukemia. Ann Thorac Surg 2018. doi: 10.1016/j.athoracsur.2017.11.014

52. Swerdlow SH, Campo E, Harris NL, et al. WHO classification of tumours of haematopoietic and lymphoid tissues. In: WHO classification of tumours, Revised 4th Edition, Volume 2; 2017.

53. Lister TA, Crowther D, Sutcliffe SB, et al. Report of a committee convened to discuss the evaluation and staging of patients with Hodgkin's disease: Cotswolds meeting. J Clin Oncol 1989. doi: 10.1200/JCO.1989.7.11.1630

54. Khorana AA, Streiff MB, Farge D, et al. Venous thromboembolism prophylaxis and treatment in cancer: A consensus statement of major guidelines panels and call to action. J Clin Oncol 2009. doi: 10.1200/JCO.2009.22.3214

55. Sanz MA, Grimwade D, Tallman MS, et al. Management of acute promyelocytic leukemia: Recommendations from an expert panel on behalf of the European LeukemiaNet. Blood 2009. doi: 10.1182/blood-2008-04-150250

56. Khorana AA, Connolly GC. Assessing risk of venous thromboembolism in the patient with cancer. J Clin Oncol 2009. doi: 10.1200/JCO.2009.22.3271

57. Di Nisio M, Ferrante N, Feragalli B, et al. Arterial thrombosis in ambulatory cancer patients treated with chemotherapy. Thromb Res 2011. doi: 10.1016/j.thromres.2010.10.001

58. Clahsen PC, Van De Velde CJH, Julien JP, et al. Thromboembolic complications after perioperative chemotherapy in women with early breast cancer: A European Organization for research and treatment of cancer breast cancer cooperative group study. J Clin Oncol 1994. doi: 10.1200/JCO.1994. 12.6.1266 
59. Levine MN, Gent M, Hirsh J, et al. The thrombogenic effect of anticancer drug therapy in women with stage II breast cancer. N Engl J Med 1988. doi: 10.1056/NEJM198802183180703

60. Falanga A, Marchetti M, Vignoli A. Coagulation and cancer: Biological and clinical aspects. J Thromb Haemost 2013. doi: 10.1111/jth.12075

61. Khorana AA, Francis CW, Culakova E, et al. Frequency, risk factors, and trends for venous thromboembolism among hospitalized cancer patients. Cancer 2007. doi: 10.1002/cncr.23062

62. Timp JF, Braekkan SK, Versteeg HH, Cannegieter SC. Epidemiology of cancer-associated venous thrombosis. Blood 2013. doi: 10.1182/blood-2013-04-460121

63. Blom JW, Doggen CJM, Osanto S, Rosendaal FR. Malignancies, prothrombotic mutations, and the risk of venous thrombosis. JAMA 2005. doi: 10.1001/jama.293.6.715

64. Konstantinides SV, Meyer G, Bueno H, et al. 2019 ESC Guidelines for the diagnosis and management of acute pulmonary embolism developed in collaboration with the European respiratory society (ERS). Eur Heart J 2020. doi:1 0.1093/eurheartj/ehz405

65. Chang H-M, Moudgil R, Scarabelli T, et al. Cardiovascular complications of cancer therapy. J Am Coll Cardiol 2017. doi: 10.1016/j.jacc.2017.09.1096

66. Reeves BN, Key NS. PL-12 acquired hemophilia in malignancy. Thrombosis Res 2012. doi: 10.1016/S0049-3848(12) 70019-1.

67. Sallah S, Wan JY, Nguyen NP, et al. Disseminated intravascular coagulation in solid tumors: Clinical and pathologic study. Thromb Haemost 2001. doi: 10.1055/s-0037-1616139

68. Kozwich DL, Kramer LC, Mielicki WP, et al. Application of cancer procoagulant as an early detection tumor marker. Cancer 1994. doi: 10.1002/1097-0142(19940815)74:4<1367::AIDCNCR2820740430>3.0.CO;2-Y

69. Molnar S, Guglielmone H, Lavarda M, et al. Procoagulant factors in patients with cancer. Hematology 2007. doi: $10.1080 / 10245330701521416$

70. Kirchhof P, Benussi S, Kotecha D, et al. 2016 ESC Guidelines for the management of atrial fibrillation developed in collaboration with EACTS. Eur Heart J 2016. doi: 10.1093 /eurheartj/ehw210
71. Holmes DR, Kar S, Price MJ, et al. Prospective randomized evaluation of the watchman left atrial appendage closure device in patients with atrial fibrillation versus long-term warfarin therapy: The PREVAIL trial. J Am Coll Cardiol 20142. doi: 10.1016/j.jacc.2014.04.029

72. Holmes DR, Reddy VY, Turi ZG, et al. Percutaneous closure of the left atrial appendage versus warfarin therapy for prevention of stroke in patients with atrial fibrillation: a randomised non-inferiority trial. Lancet 2009. doi: 10.1016/S01406736(09)61343-X

73. Hildick-Smith D, Landmesser U, Camm AJ, et al. Left atrial appendage occlusion with the Amplatzer ${ }^{\mathrm{TM}}$ Amulet ${ }^{\mathrm{TM}}$ device: full results of the prospective global observational study. Eur Heart J 2020. doi: 10.1093/eurheartj/ehaa169

74. Hennekens CH, Dyken ML, Fuster V. Aspirin as a therapeutic agent in cardiovascular disease: A statement for healthcare professionals from the American heart association. Circulation 1997. doi: 10.1161/01.CIR.96.8.2751

75. Berger JS, Brown DL, Becker RC. Low-dose aspirin in patients with stable cardiovascular disease: A meta-analysis. Am J Med 2008. doi: 10.1016/j.amjmed.2007.10.002

76. Landolfi R, Marchioli R, Kutti J, et al. Efficacy and safety of low-dose aspirin in polycythemia vera. N Engl J Med 2004. doi: 10.1056/NEJMoa035572

77. Streiff MB, Holmstrom B, Ashrani A, et al. Cancer-associated venous thromboembolic disease, Version 1.2015. J Natl Compr Cancer Netw 2015. doi: 10.6004/jnccn.2015.0133

78. Agnelli G, Gussoni G, Bianchini C, et al. Nadroparin for the prevention of thromboembolic events in ambulatory patients with metastatic or locally advanced solid cancer receiving chemotherapy: a randomised, placebo-controlled, double-blind study. Lancet Oncol 2009. doi:10.1016/S1470-2045(09) 70232-3

79. Kaplan E. Venous thromboembolic disease. In: C.J. Wong, N.P. Hamli, editors. The perioperative medicine consult handbook. New York: Springer. 2015.

80. Khorana A, Kuderer NM, Culakova E, et al. Development and validation of a predictive model for chemotherapy- associated thrombosis. Blood 2008. doi: 10.1182/blood-2007-10-116327 